\title{
Noninvasive Monitoring of Patients With Chronic Hepatitis C
}

\author{
Robert J. Fontana and Anna S. F. Lok
}

\begin{abstract}
Hepatic fibrosis is the main determinant of clinical outcomes of chronic hepatitis C. Liver histology is frequently considered the gold standard for assessing hepatic fibrosis. However, liver biopsy is associated with sampling error, interobserver variability, and potential complications. Thus, there is a need for simple, inexpensive, and reliable noninvasive means to assess disease severity in patients with chronic hepatitis $\mathrm{C}$. Clinical examination is unreliable in differentiating different stages of compensated liver disease. Among the routine laboratory tests, decreased platelet count, increase in the ratio of aspartate to alanine aminotransferase (AST/ALT), and prolonged prothrombin time are the earliest indicators of cirrhosis and portal hypertension. Individual serum fibrosis markers have limited accuracy in predicting hepatic fibrosis. Indices composed of a panel of markers correlate better with histological fibrosis, but their reliability requires further validation. Currently, noninvasive monitoring of patients with chronic hepatitis $\mathrm{C}$ relies on clinical evaluation, routine laboratory tests, and ultrasound and endoscopic surveillance in patients with cirrhosis. Initial evaluation should focus on assessment of activity and stage of liver disease for prognostication and decisions regarding treatment, and to rule out coinfections and other causes of liver disease. Subsequent follow-up should focus on detection of liver disease progression and the need for treatment. The frequency of monitoring and the tests used will depend on the patient's age, stage of liver disease, and comorbid conditions. There is an urgent need to develop and validate noninvasive tests that can accurately reflect the full spectrum of hepatic inflammation and fibrosis in chronic hepatitis C. (HEPATOLOGY 2002;36:S57-S64.)
\end{abstract}

A $\mathrm{n}$ important component of management of hepatitis $\mathrm{C}$ is the clinical assessment of disease severity. Liver histology is frequently considered the gold standard for establishing the severity of hepatic necroinflammation and fibrosis. However, liver biopsy is associated with risks of complications, patient discomfort, and expense. Therefore, tracking of liver disease progression

Abbreviations: $A S T$, aspartate aminotransferase; $A L T$, alanine aminotransferase; HCV, hepatitis C virus; PIIINP, $N$-terminal propeptide of type III collagen; TIMP-1, tissue inbibitory metalloprotease-1; MMP, matrix metalloproteinase.

From the Division of Gastroenterology, University of Michigan Health System. Ann Arbor, MI.

Drs. Fontana and Lok are supported in part by National Institutes of Health contract N01-DK-92323 and grants U01-DK-60344 and U01-DK-57577. Dr. Fontana has received research grant support from and is on the speakers' bureau for Schering-Plough Research Institute, Hoffmann-La Roche, and GlaxoSmithKline. Dr. Lok has received research grant support from Schering-Plough, Hoffmann-La Roche, Bristol-Myers Squibb, Gilead Sciences, and GlaxoSmithKline. Dr. Lok is on the advisory board or serves as a consultant for Idenix, Gilead Sciences, GlaxoSmithKline, and Chiron Corporation.

Address reprint requests to Anna S. F. Lok, M.D., Division of Gastroenterology, University of Michigan Medical Center, 3912 Taubman Center, Ann Arbor, MI 48109-0362.E-mail: aslok@umich.edu; fax: 734-936-7392.

Copyright (C) 2002 by the American Association for the Study of Liver Diseases. 0270-9139/02/3605-1007\$35.00/0

doi:10.1053/jhep.2002.36800 cannot rely on repeated biopsies. In addition, interobserver variability and sampling error may lead to erroneous staging. For example, studies involving expert hepatopathologists showed an interobserver agreement in fibrosis staging ranging from $70 \%$ to $90 \%$ and an intraobserver agreement of $60 \%$ to $90 \% .^{1,2}$ The level of agreement for necroinflammation was uniformly lower. Studies comparing needle biopsy specimens with laparoscopic examination of the liver reported an underestimation of cirrhosis in $15 \%$ to $30 \%$ of patients, while studies of multiple liver biopsies obtained from the right lobe of the liver showed a sampling error of $10 \%$ to $20 \% .3,4$ These findings are not surprising because a $2 \mathrm{~cm}$ core of liver tissue represents only $1 / 50,000$ th of the entire organ.

A noninvasive means of assessment of disease activity and stage would be very helpful, particularly in monitoring patients with chronic hepatitis $\mathrm{C}$ virus (HCV) infection over time. An ideal test would be simple, readily available, inexpensive, accurate, and sensitive to the effects of therapy (Table 1). At present, the available noninvasive means of assessing liver disease severity and stage are imperfect and lack accuracy and reliability. Noninvasive approaches to assessment of severity of hepatitis $\mathrm{C}$ 
Table 1. Criteria for Ideal Noninvasive Tests for Monitoring Patients With Chronic Hepatitis C

Simple

Readily available

Inexpensive

Accurate prediction of the full spectrum of liver disease

Reproducible

Sensitive to the effects of treatment

Useful in tracking disease progression

include clinical symptoms and signs, routine biochemical and hematologic blood tests, serum markers of fibrosis and inflammation, combinations of clinical and blood test results, quantitative assays of hepatic function, and radiologic imaging studies (Table 2 ).

\section{Clinical Features of Disease Severity}

Clinical symptoms and signs of liver disease are often unreliable in assessing disease severity in patients with compensated liver disease. A variety of symptoms including fatigue, itching, and right upper abdominal discomfort, as well as impaired quality of life, occur in patients with chronic hepatitis $\mathrm{C}$, but symptoms and quality of life scores correlate poorly with liver disease severity. Likewise, physical findings of chronic liver disease, such as jaundice, pedal edema, hepatosplenomegaly, ascites, and encephalopathy, are frequently absent until patients develop decompensated cirrhosis.

Adverse outcomes of chronic hepatitis $\mathrm{C}$ including liver failure, hepatocellular carcinoma, and death rarely occur in pre-cirrhotic patients. Despite improvements in the efficacy of treatment of chronic hepatitis C, therapy is not recommended for all patients because of the indolent course in some patients, and the expense, unpleasant side effects, and low overall efficacy (50\% to $60 \%$ ) of current treatment. Thus, accurate determination of the extent of hepatic fibrosis or stage of liver disease is important for prognostication, decisions regarding treatment, and monitoring of disease progression.

Although clinical and historical data have been shown to correlate with rate of fibrosis progression, their accuracy in predicting stage of frbrosis on liver biopsies is low. In a study of 2,235 French patients with chronic hepatitis C, Poynard et al ${ }^{5}$ identified 3 independent factors associated with an increased rate of hepatic fibrosis: age at infection older than 40 years, daily alcohol consumption of $50 \mathrm{~g}$ or more, and male sex. However, the same investigators found that the discriminatory value of these 3 clinical factors for significant (Metavir score F2 to F4) as well as advanced fibrosis (Metavir score F3 and F4) was inferior compared with a 5-marker biochemical index (vide infra) (T. Poynard, personal communications, April 2002). A poor correlation between estimated fibrosis based on the 3 historical factors listed previously and histological fibrosis was also observed in a study of 106 Canadian patients. ${ }^{6}$ Thus, clinical and historical data alone cannot reliably predict hepatic fibrosis in patients with chronic hepatitis C. Other clinical factors that have been shown to be associated with more severe or rapidly progressive liver disease include coinfection with hepatitis B virus or human immunodeficiency virus, and long-term immunosuppressive therapy. 7,8

\section{Routine Laboratory Tests}

Laboratory tests that are routinely included in the evaluation of patients with hepatitis $C$ include a serum panel of liver tests (albumin, total bilirubin, aspartate aminotransferase $[\mathrm{AST}]$, alanine aminotransferase [ALT], and alkaline phosphatase), prothrombin time, and complete blood counts. Serum ALT levels reflect liver injury, but the correlation between ALT levels and necroinflammatory and fibrosis scores is poor. ${ }^{7,8}$ Despite the poor quantitative correlation, most patients with persistently normal ALT levels (at least 3 normal values over a 6 to 12 month period) have less inflammation and fibrosis ${ }^{9-11}$ and slower rate of fibrosis progression compared with patients with elevated ALT levels. One study found that AST had a stronger correlation with liver histology, in particular hepatic fibrosis. ${ }^{11}$ Reversal of AST/ALT ratio was observed when patients progress from chronic hepatitis to cirrhosis $^{12}$ and AST/ALT ratio of more than 1 had been reported to have very high specificity ( $82 \%$ to $100 \%)$ for cirrhosis. ${ }^{13-16}$ Serum albumin, bilirubin, and prothrombin time reflect hepatic function, but these values frequently remain normal in patients with compensated or early cirrhosis.

Among the routine laboratory tests, decreased platelet count, reversal of AST/ALT ratio, and prolonged prothrombin time are the earliest indicators of cirrhosis. ${ }^{15-22}$ A study of 323 patients with chronic hepatitis $C$ found that platelet count or prothrombin time tested at a single time point was predictive of significant hepatic fibrosis (Metavir score F2 to F4 [T. Poynard et al., personal com-

\section{Table 2. Noninvasive Methods to Monitor Patients With} Chronic Hepatitis C

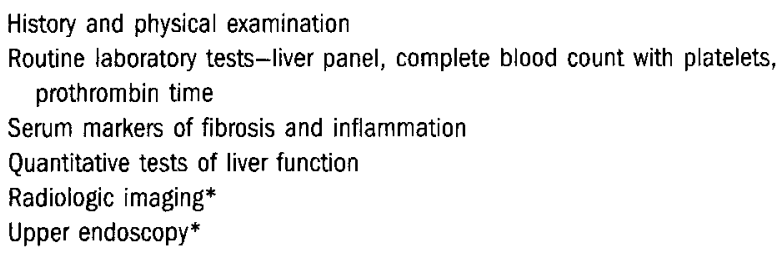

*For patients with cirrhosis or significant fibrosis. 
Table 3. Serum Markers of Liver Fibrosis

\begin{tabular}{|c|c|c|}
\hline Markers & Clearance & Observations \\
\hline \multicolumn{3}{|c|}{ Products of collagen synthesis/degradation } \\
\hline PIIINP & Hepatic and renal & $\begin{array}{l}\text { Marker of early fibrogenesis and inflammation, correlate better } \\
\text { with histologic inflammation than fibrosis, levels decline with } \\
\text { interferon therapy }\end{array}$ \\
\hline Type IV collagen & Hepatic and renal & Marker of fibrolysis; levels decline with IFN therapy \\
\hline \multicolumn{3}{|l|}{ Extracellular matrix enzymes } \\
\hline TIMP-1 & Intravascular & $\begin{array}{l}\text { Circulating inhibitors of MMP enzymes that can enhance } \\
\text { fibrogenesis (TIMP-1); rapidly cleared from serum }\end{array}$ \\
\hline MMP & Intravascular & $\begin{array}{l}\text { MMP's degrade extracellular matrix, weak correlation with } \\
\text { fibrosis stage }\end{array}$ \\
\hline \multicolumn{3}{|l|}{ Extracellular matrix components } \\
\hline Hyaluronic acid & Hepatic endothelium and renal & $\begin{array}{l}\text { Glycosaminoglycan produced by stellate cells, levels increased } \\
\text { in patients with cirrhosis, levels decline with IFN therapy }\end{array}$ \\
\hline Laminin & Hepatic endothelium & $\begin{array}{l}\text { Basement membrane glycoprotein; levels may reflect severity of } \\
\text { portal hypertension }\end{array}$ \\
\hline \multicolumn{3}{|l|}{ Others } \\
\hline Transforming growth factor-beta & Hepatic/intravascular & $\begin{array}{l}\text { Marker of fibrogenesis, Single most important cytokine involved } \\
\text { in stellate cell activation and proliferation, presence in } \\
\text { platelets makes accurate measurement difficult, levels } \\
\text { decline with interferon therapy }\end{array}$ \\
\hline YKL-40 & ? Hepatic & $\begin{array}{l}38 \text { kd glycoprotein produced by stellate cells; levels increased } \\
\text { in patients with moderate fibrosis, sensitive to fibrosis } \\
\text { progression over time }\end{array}$ \\
\hline
\end{tabular}

munication, April 2002]). Preliminary analysis of the baseline data of 667 hepatitis $C$ patients enrolled in the HALT-C (Hepatitis C Antiviral Long-term Treatment against Cirrhosis) trial found that a multivariate logistic regression model comprising platelet count, AST/ALT ratio, alkaline phosphatase, and prothrombin time was predictive of cirrhosis. ${ }^{23}$ In other studies of patients with chronic liver disease undergoing surveillance endoscopy, a low platelet count and prolonged prothrombin time correlated with the presence and size of esophageal varices, but the specificity for the presence of varices was low $(58 \%) .{ }^{24}$ Other investigators have also proposed that a composite index that includes platelet count and serum bilirubin levels may be a useful means of identifying patients with $\mathrm{HCV}$-related cirrhosis who should undergo surveillance for hepatocellular carcinoma. ${ }^{25}$ These studies suggest that combinations of routine laboratory tests may be used to predict significant fibrosis or cirrhosis in chronic hepatitis $\mathrm{C}$ patients, but the sensitivity and specificity of these tests is suboptimal ( $70 \%$ to $80 \%$ at best). In addition, the validity of these tests/models in the general population of hepatitis $\mathrm{C}$ patients remains to be determined.

\section{Serum Markers of Fibrosis and Inflammation}

In attempts to improve the accuracy of predicting histological activity and fibrosis, many investigators have explored the use of serum markers of inflammation and fibrosis. Ideally, serum markers of inflammation and fibrosis should be simple, inexpensive, readily available, reproducible, and accurate. Potential markers of fibrosis include products of collagen synthesis or degradation, enzymes involved in matrix biosynthesis or degradation; extracellular matrix glycoproteins, and proteoglycans/glycosaminoglycans (Table 3). For these markers to accurately reflect hepatic fibrogenesis or fibrolysis, they should be organ-specific and the biological half-life should be independent of urinary and biliary excretion as well as sinusoidal endothelial uptake. Unfortunately, none of the available markers fulfill all these criteria.

Serum hyaluronic acid and N-terminal propeptide of type III collagen (PIIINP) levels have been most extensively evaluated in patients with chronic hepatitis C. Increase in serum hyaluronic acid level may be a result of increased hepatic stellate cell production as well as a decrease in removal by hepatic sinusoidal endothelial cells. Several studies showed that serum hyaluronic acid levels correlate with the degree of hepatic fibrosis in patients with chronic hepatitis $C .^{26,27}$ The primary role of testing for serum hyaluronic acid level is to identify patients without cirrhosis (greater than $90 \%$ negative predictive value). One study of 486 chronic hepatitis $\mathrm{C}$ patients found that a serum hyaluronic acid level of less than $60 \mu \mathrm{g} / \mathrm{L}$ had $99 \%$ accuracy in predicting absence of cirrhosis on liver histology (negative predictive value), but the accuracy of serum hyaluronic acid level in diagnosing cirrhosis was low $\left(30 \%\right.$ positive predictive value). ${ }^{26}$ Serum levels of 
PIIINP correlate better with inflammation and are inferior to serum hyaluronic acid levels in predicting hepatic fibrosis. ${ }^{27}$ Recently, serum YKL-40 has been reported to be superior to hyaluronic acid levels in predicting hepatic fibrosis. ${ }^{28,29}$ YKL-40 is a mammalian member of a chitinase family with a molecular weight of $40 \mathrm{kd}$. The physiologic functions of YKL- 40 are unknown. The protein is expressed in human liver and may be involved in the remodeling of the extracellular matrix. A recent study of 129 patients with different causes of chronic liver disease found that serum YKL- 40 levels were increased, even in patients with mild fibrosis, and correlated better with histological fibrosis scores than serum hyaluronic acid levels. ${ }^{28,29}$ Further studies in larger numbers of patients are needed to confirm the utility of testing for YKL-40 levels in assessing hepatic fibrosis in patients with chronic hepatitis $\mathrm{C}$.

Based on available data, it is unlikely that individual serum markers of fibrosis can replace liver histology. Whether a combination of markers will have better accuracy remains to be determined. In an ongoing study in Europe, a panel of 10 markers were evaluated; PIIINP, hyaluronic acid, tissue inhibitory metalloprotease- 1 (TIMP-1), and collagen IV correlated best with histological fibrosis scores. ${ }^{30}$ The final results of this study are awaited.

Other investigators have studied a combination of routine laboratory tests and serum markers of fibrosis and inflammation. One study of 339 patients with chronic hepatitis $\mathrm{C}$ found that an index based on patient age and gender combined with 5 biochemical markers (total bilirubin, haptoglobin, gamma glutamyl transpeptidase, alpha- 2 macroglobulin, and apolipoprotein A1) was the most accurate in identifying patients with significant hepatic fibrosis. ${ }^{31}$ An index of 0 to 0.10 had a $100 \%$ negative predictive value, while an index of 0.60 to 1.00 had a greater than $90 \%$ positive predictive value for a Metavir score of F2 to F4. Inclusion of other markers did not improve the predictive value. Subsequent studies from the same investigators found that the 5-marker index was superior to historical data (age at infection and alcohol consumption), platelet count, or prothrombin time in predicting significant fibrosis (Metavir score of F2 to F4) or advanced fibrosis (F3 and F4). These findings are very encouraging but need to be validated in other laboratories and other patient populations. A major limiting factor in the use of this index is that $50 \%$ of the patients fall into the intermediate zone (index 0.20 to 0.80 ) where the predictive accuracy is low. In addition, the index was designed to detect the presence of clinically significant fibrosis. It has a low negative predictive value for mild fibrosis. Thus, the ability to differentiate among varying degrees of hepatic fibrosis is limited. Furthermore, all except one study on the 5-marker index were cross-sectional and the utility of the index in monitoring disease progression remains to be determined.

\section{Quantitative Tests of Liver Function}

Quantitative tests of liver function are based on the turnover of a test substance, which is metabolized almost exclusively by the liver. Clearance of the substrate is determined by hepatic blood flow (hepatic artery and portal vein) and hepatic metabolic capacity. A variety of oral and intravenously administered metabolic probes, such as aminopyrine, galactose, and monoethyl glycine xylidide, have been evaluated. In general, these metabolic tests can reliably differentiate patients with compensated versus decompensated cirrhosis. Tests of hepatic metabolism and tests of hepatic blood flow (indocyanine green clearance) have been used mainly in the prediction of survival of patients with cirrhosis, especially after surgery, ${ }^{32}$ but their ability to identify patients with mild or moderate fibrosis has not been properly examined.

A recent study compared 2 tests of hepatic metabolism (aminopyrine breath test and galactose elimination capacity) and 2 tests of hepatic blood flow (indocyanine green clearance and sorbitol clearance) in 367 patients with chronic hepatitis B or $\mathrm{C}$, and 25 age-matched healthy controls. ${ }^{33}$ The investigators found that metabolic liver function was significantly decreased even in patients with mild or moderate fibrosis. In contrast, hepatic perfusion declined only in patients with severe fibrosis or cirrhosis. These data suggest that tests of hepatic metabolic function may be used in assessing hepatic fibrosis. However, these tests are cumbersome and expensive and unlikely to be applicable in clinical practice. In addition, the performance (predictive values for different stages of fibrosis) of these tests was not analyzed and their accuracy in comparison with simpler more readily available tests was not discussed.

\section{Radiologic Imaging}

Ultrasound and other imaging techniques such as computed tomography and magnetic resonance imaging can be used to diagnose cirrhosis based on the presence of an enlarged spleen, small nodular liver, ascites, and intraabdominal varices. In addition, radiologic imaging can detect hepatocellular carcinoma. However, ultrasound is highly operator-dependent while computed tomography and magnetic resonance imaging are very expensive. In addition, the presence of increased liver echogenicity on ultrasound does not reliably differentiate hepatic steatosis from fibrosis. Furthermore, current imaging techniques 
are unable to assess the extent of hepatic fibrosis and are not sufficiently sensitive to detect early cirrhosis. A recent study examined 12 clinical and 11 Doppler ultrasonic variables in 243 patients with chronic (alcoholic and viral) liver disease among whom $45 \%$ had evidence of clinical decompensation (ascites or encephalopathy). The diagnostic accuracy of Doppler ultrasound for cirrhosis among the 83 patients with chronic hepatitis was $86 \%$ (positive predictive value, 62\%; negative predictive value, 92\%). ${ }^{34}$ Nodularity on the liver surface was the most discriminatory ultrasound feature. These data suggest that cirrhosis can be diagnosed by ultrasound in $60 \%$ to $80 \%$ of patients in the best of hands. However, the generalizability of these data to clinical practice is uncertain. In addition, the investigators acknowledged that not all patients in the study could be evaluated because of anatomic limitations. Recently, contrast-enhanced Doppler ultrasonography has also been reported to increase the accuracy of the diagnosis of cirrhosis. ${ }^{35}$ These data are preliminary and require confirmation by other groups.

Radionuclide liver spleen scans using technetium $99 \mathrm{~m}$-labeled sulphur colloid taken up by Kupffer cells have also been used to assess hepatic function. ${ }^{36}$ This technique is sensitive in diagnosing cirrhosis, but there is very little data on its accuracy in detecting mild or moderate hepatic fibrosis. The expense of this test and its low sensitivity in detecting early fibrosis limit its clinical use in patients with chronic hepatitis $\mathrm{C}$.

\section{Recommendations on Noninvasive Monitoring}

Significant progress has been made in the noninvasive assessment of hepatic fibrosis. However, none of the tests or indices discussed previously will, on their own, replace liver histology as a means of assessing activity and stage of disease in the near future. Additional refinement and validation of these tests and indices are necessary to confirm their applicability in clinical practice and their value, not only in the initial assessment of patients, but also in monitoring disease progress. Ideally, these tests and indices should provide accurate prediction of the full spectrum of liver disease, not just dichotomous outcomes. Noninvasive tests that could reliably distinguish moderate to severe fibrosis from mild fibrosis may also prove useful to clinicians for treatment decisions and disease monitoring. Recommendations on noninvasive monitoring of patients with chronic hepatitis $C$ in 2002 will continue to rely on clinical evaluation, routine laboratory tests, and standard imaging. The reader should be aware that the following recommendations have not been validated in prospective studies. Because the appropriate monitoring plan will vary in light of the relevant facts and circum-
Table 4. Initial Noninvasive Evaluation of Patients With Chronic Hepatitis C*

History
Symptoms
Risk factors for hepatitis C, age at infection
Alcohol consumption
Comorbid illnesses
Physical examination
Blood tests
HCV tests-HCV RNA, HCV genotype
Tests to rule out HBV and HIV coinfection and other cause of liver disease
Tests to assess liver disease severity-liver panel, blood counts with platelets,
prothrombin time
Test to detect hepatocellular carcinoma-alpha-fetoprotein
Abdominal ultrasound

* Baseline liver biopsy is useful in patients above the age of 40 , patients who wish to defer treatment, and patients with relative contraindications to treatment.

stances surrounding each individual patient with chronic hepatitis $\mathrm{C}$, these recommendations are intended to serve as guidelines and not "standards of care," and may be updated as new information becomes available.

\section{Initial Evaluation}

The initial evaluation of patients with chronic hepatitis C should focus on assessment of activity and stage of liver disease for prognostication, decisions regarding treatment, and to rule out other causes of liver disease (Table 4). Patients should undergo a thorough history and physical examination to estimate the age at infection, quantify alcohol consumption, and to evaluate symptoms and signs of chronic liver disease.

A sensitive test for HCV RNA is recommended in patients with antibody to $\mathrm{HCV}$ (anti-HCV) who have normal ALT levels and no risk factors for infection, because up to $30 \%$ of these individuals may have resolved infection or a false-positive anti-HCV test result. In contrast, the pretest probability is high in anti-HCV-positive patients with risk factors and/or clinical or biochemical evidence of chronic liver disease. Therefore, a confirmatory test for HCV RNA is not mandatory but is highly recommended because patients with risk factors may have resolved HCV infection and other causes of aminotransferase elevations (e.g., fatty liver disease, alcohol). Quantitative HCV RNA levels and HCV genotypes do not correlate with disease severity, but these tests should be performed in patients who are considered for therapy because the results are useful in predicting the likelihood of an antiviral treatment response and in planning the duration of therapy.

The initial laboratory tests should include a liver panel, complete blood counts with platelet counts, prothrombin time, and alpha-fetoprotein. As discussed previously, decreased platelet count, reversal of AST/ALT ratio, and 
Table 5. Long-Term Noninvasive Monitoring of Patients With Chronic Hepatitis C

\begin{tabular}{|c|c|c|c|}
\hline & \multirow[b]{2}{*}{$\begin{array}{l}\text { Persistently } \\
\text { Normal ALT }\end{array}$} & \multicolumn{2}{|c|}{ Abnormal ALT } \\
\hline & & $\begin{array}{c}\text { Mild } \\
\text { Liver } \\
\text { Disease }\end{array}$ & $\begin{array}{c}\text { Significant } \\
\text { Fibrosis or } \\
\text { Clrrhosis }\end{array}$ \\
\hline Frequency & 6 to $12 \mathrm{mos}$ & $6 \mathrm{mos}$ & $6 \mathrm{mos}$ \\
\hline History and physical & + & + & + \\
\hline Routine lab tests & + & + & + \\
\hline Alpha-fetoprotein & - & - & + \\
\hline Ultrasound & - & - & + \\
\hline Upper endoscopy & - & - & $t^{*}$ \\
\hline
\end{tabular}

* Every 2 years.

prolongation of prothrombin time indicate that the patient may have advanced fibrosis or cirrhosis. Laboratory tests at presentation should also include tests for other causes of liver disease such as hemochromatosis (iron saturation) and hepatitis B (hepatitis B surface antigen) and tests for antibody to human immunodeficiency virus in patients with risk factors. A baseline abdominal ultrasound should be considered, particularly for patients above the age of 40 , to determine if there are features suggestive of cirrhosis and to rule out focal lesions in the liver. Assessment of underlying autoimmune disorders, thyroid dysfunction, neuropsychiatric illnesses, and coronary artery disease should be carried out before initiation of treatment.

Despite the shortcomings of liver biopsies, a baseline liver biopsy may be very useful, especially in patients above the age of 40 and patients who wish to defer treatment.

Monitoring of treatment response and adverse effects will be discussed in detail in the sections on treatment.

\section{Long-Term Follow-Up}

The frequency at which hepatitis $\mathrm{C}$ patients should be monitored and the tests that should be performed depend on the age of the patients, stage of liver disease, and comorbid conditions (Table 5). Decisions regarding treatment as well as re-treatment should be re-evaluated in patients who have had progression of liver disease, resolution of contraindications to treatment, improvement, or stabilization of comorbid illnesses. Re-evaluation is also needed as each better-tolerated and more effective treatments become available.

Patients with detectable serum HCV RNA, persistently normal ALT levels and no clinical, laboratory, and radiologic evidence suggestive of cirrhosis may be monitored at less frequent intervals. Clinical evaluation and routine laboratory tests at 6 - to 12 -month intervals will suffice. More frequent monitoring and evaluation into other causes of liver disease should be initiated in patients who develop abnormal ALT levels during the course of follow-up.

Patients with abnormal ALT levels who are considered to have mild liver disease should undergo clinical assessment and routine laboratory tests at 6 -month intervals. The focus of noninvasive monitoring is to detect signs of liver disease progression. Monitoring is particularly important for patients who choose to defer treatment based on the finding of mild liver disease at presentation. Because of the poor sensitivity and specificity of clinical examination and routine laboratory tests, an abdominal ultrasound and possibly a liver biopsy should be considered in patients who have clinical and/or laboratory evidence of liver disease progression.

Patients with abnormal ALT levels who have a high probability of significant hepatic fibrosis or cirrhosis require more intense monitoring to detect early signs of portal hypertension, hepatic decompensation, and hepatocellular carcinoma. Thorough clinical evaluation and routine laboratory tests should be performed at 6-month intervals. Although the efficacy of alpha-fetoprotein and ultrasound in the surveillance of hepatocellular carcinoma has not yet been proven, these tests are often included in the 6-month evaluation of hepatitis $\mathrm{C}$ patients who have cirrhosis or advanced fibrosis. In addition, surveillance upper endoscopy for patients with early cirrhosis is recommended to detect esophageal varices and other stigmata of portal hypertension at 2-year intervals. ${ }^{37}$

\section{Future Research Needs}

Significant progress has been made in the development of noninvasive tests for the monitoring of patients with chronic hepatitis C. Nevertheless, none of the currently available tests can completely replace liver histology. Because of the variable course of chronic hepatitis $C$, and the complexity of host, virus, and environmental factors that may influence liver disease progression, availability of noninvasive tests that can be used repeatedly to monitor patients with chronic hepatitis $\mathrm{C}$ is crucial. These tests should be simple, inexpensive, readily available, and capable of accurate reflection of the entire spectrum of hepatic inflammation and fibrosis in the general population of hepatitis $C$ patients. It is likely that no single test will fulfill all these criteria. Nevertheless, research to identify new serum markers that correlate with hepatic inflammation and fibrosis should continue. In addition, studies evaluating indices composed of readily available, standardized laboratory tests (with or without simple demographic data such as age and gender) should be encouraged. Indices that show promise need to be validated using several laboratories and patient populations, 
and should be accurate, not only in assessing liver disease at initial evaluation but also in tracking disease progression during follow-up. These indices should also be sensitive to the effects of treatment. For these goals to be accomplished, clinical centers and basic science laboratories will need to work together. Studies of noninvasive markers should include adequate numbers of patients of both sexes and a wide range of ages and ethnicities who have the full spectrum of liver disease and who are followed for a sufficient duration of time to capture disease progression. Existing clinical databases and serum repositories from well-characterized patients with hepatitis C patients may provide materials for preliminary analysis of candidate indices. Prospective multicenter studies involving a broader spectrum of patients will be necessary to validate these indices. Based on the progress of the last 10 years, there is every reason to believe that accurate noninvasive monitoring of patients with chronic hepatitis $C$ can become a reality in the next decade.

\section{References}

1. Bedossa P, Poynard T and the Metavir Cooperative Group. Intraobserver and interobserver variations in liver biopsy interpretation in patients with chronic hepatitis C. HepaTOLOGY 1994;20:15-20.

2. Westin J, Lagging LM, Wejstal R, Norkans G, Dhillon AP. Interobserver study of liver histopathology using the Ishak score in patients with chronic hepatitis C virus infection. Liver 1999;19:183-187.

3. Poniachik J, Bernstein DE, Reddy R, Jeffers LJ, Coelho-Little M, Civantos F, Schiff ER. The role of lapraoscopy in the diagnosis of cirrhosis. Gastrointest Endosc 1996;43:568-571.

4. Maharaj B, Maharaj RJ, Leary WP, Cooppan RM, Naran AD, Pirie D, Pudifin DJ. Sampling variability and its influence on the diagnostic yield of percutaneous needle biopsy of the liver. Lancet 1986;327:523-525.

5. Poynard T, Bedossa P, Opolon P. Natural history of liver fibrosis progression in patients with chronic hepatitis C. The OBSVIRC, METAVIR, CLINIVIR, and DOSVIRC groups. Lancet 1997;349:825-832.

6. Myers RP, Hilsden RJ, Lee SS. Historical features are poor predictors of liver fibrosis in Canadian patients with chronic hepatitis C. J Viral Hepat 2001;8:249-255.

7. Zarkki JP, Bohn B, Bastie A, Pawlotsky JM, Baud M, Bost-Bezeaux F, van Nhieu JT, et al. Characteristics of patients with dual infection by hepatitis $B$ and $C$ viruses. J Hepatol 1998;28:27-33.

8. Benhamou Y, Bochet M, Di Martino V, Charlotte F, Azria F, Coutelier A, Vidaud M, et al. for the MULTIVIRC Group. Liver fibrosis progression in human immunodeficiency virus and hepatitis $\mathrm{C}$ virus coinfected patients. Hepatology 1999;30:1054-1058.

9. McCormick SE, Goodman ZD, Maydonovitch CL, Sjogren MH. Evaluation of liver histology, ALT elevation, and HCV RNA titer in patients with chronic hepatitis C. Am J Gastroenterol 1996;91:1516-1522.

10. Haber MM, West AB, Haber AD, Reuben A. Relationship of aminotransferases to liver histological status in chronic hepatitis $\mathrm{C}$. Am J Gastroenterol 1995;90:1250-1257.

11. Gordon SC, Fang JW, Silverman AL, McHutchison JG, Albrecht, JK. The significance of baseline serum alanine aminotransferase on pretreatment disease characteristics and response to antiviral therapy in chronic hepatitis C. Hepatology 2000;32:400-404.

12. Persico M, Persico E, Suozzo R, Conte S, De Seta M, Coppola L, Palmentieri $B$, et al. Natural history of hepatitis $C$ virus carriers with persistently normal aminotransferase levels. Gastroenterology 2000;1 18:760-764.
13. Mathurin P, Moussalli J, Cadranel JF, Thibault V, Charlotte F, Dumouchel P, Cazier A, et al. Slow progression rate of fibrosis in hepatitis C virus patients with persistently normal alanine transaminase activity. Hepatology 1998;27:868-872.

14. Assy N, Minuk GY. Serum aspartate but not alanine aminotransferase levels help to predict the histological features of chronic hepatitis $\mathrm{C}$ viral infections in adults. Am J Gastroenterol 2000;95:1545-1550.

15. Williams AL, Hoofnagle JH. Ratio of serum aspartate to alanine aminotransferase in chronic hepatitis. Relationship to cirrhosis. Gastroenterology 1988;95:734-739.

16. Park GJ, Lin BP, Ngu MC, Jones, DB, Katelaris PH. Aspartate aminotransferase: alanine aminotransferase ratio in chronic hepatitis $\mathrm{C}$ infection: is it a useful predictor of cirrhosis? ] Gastroenterol Hepatol 2000;15:386390

17. Imperiale TF, Said AT, Cummings OW, Born LJ. Need for validation of clinical decision aids: use of the AST/ALT ratio in predicting cirrhosis in chronic hepatitis C. Am J Gastroenterol 2000;95:2328-2332.

18. Pohl A, Behling C, Oliver D, Kilani M, Monson P, Hassanein T. Serum aminotransferase levels and platelet counts as predictors of degree of fibrosis in chronic hepatitis C virus infection. Am J Gastroenterol 2001;96: 3142-3146.

19. Sheth S, Flamm S, Gordon F, Chopra S. AST/ALT ratio predicts cirrhosis in patients with chronic hepatitis $\mathrm{C}$ virus infection. Am J Gastroenterol 1998;93:44-48.

20. Poynard T, Bedossa P. Age and platelet count: a simple index for predicting the presence of histological lesions in patients with antibodies to hepatitis C virus. METAVIR and CLINIVIR Cooperative Study Groups. J Viral Hepatitis 1997;4:199-208.

21. Marsumura H, Moriyama M, Goto I, Tanaka N, Okubo H, Arakawa Y. Natural course of progression of liver fibrosis in Japanese patients with chronic liver disease type C-a study of 527 patients at one establishment. J Viral Hepatitis 2000;7:268-275.

22. Oberti F, Valsesia E, Pilette C, Rousselet MC, Bedossa P, Aube C, Gallois $Y$, et al. Noninvasive diagnosis of hepatic fibrosis or cirrhosis. Gastroenterology 1997;113:1609-1616.

23. Lok AS, Everhart J, Everson G, Wright E, Sterling R, Ghany MG, Goodman $Z$, and the HALT-C trial investigators. Clinical model to predict cirrhosis in patients with fibrotic stages of chronic hepatitis $\mathrm{C}$ : an analysis of the HALT-C patient population. HEPATOLOGY 2002 (in press).

24. Pilette C, Oberti F, Aube C, Rousseler MC, Bedossa P, Gallois Y, Rifflet H, et al. Non-invasive diagnosis of esophageal varices in chronic liver diseases. J Hepatol 1999;31:867-873.

25. Degos F, Christidis C, Ganne-Carrie N, Farmachidid JP, Degott C, Guettier $\mathrm{C}$, Trinchet JC, et al. Hepatitis $\mathrm{C}$ virus related cirrhosis: time to occurrence of hepatocellular carcinoma and death. Gut 2000;47:131-136.

26. McHutchison JG, Blatt LM, de Medina M, Craig JR, Conrad A, Schiff ER, Tong MJ. Measurement of serum hyaluronic acid in patients with chronic hepatitis $\mathrm{C}$ and its relationship to liver histology. Consensus Interferon Study Group. J Gastroenterol Hepatol 2000;15:945-951.

27. Guechot J, Laudat A, Loria A, Serfaty L, Poupon R, Giboudeau J. Diagnosric accuracy of hyaluronan and type III procollagen amino-terminal peptide serum assays as markers of liver fibrosis in chronic viral hepatitis $\mathrm{C}$ evaluated by ROC curve analysis. Clin Chem 1996;42:558-563.

28. Johansen JS, Christoffersen P, Moller S, Price PA, Henriksen JH, Garbarsch C, Bendtsen F. Serum YKL-40 is increased in patients with hepatic fibrosis. J Hepatol 2000;32:911-920.

29. Kamal SM, Turner B, Koziel MJ, Afdhal NH. YKL-40 and PIIINP correlate with the progression of fibrosis in chronic hepatitis $\mathrm{C}$ [Abstract]. Gastroenterology 2001;120:1895A.

30. Rosenberg W, Burt A, Hubscher S, Roskams T, Voekler M, Becka M, Arthur MJ. Serum markers predict liver fibrosis [Abstract]. HEPATOLOGY 2001;34:396A.

31. Imberr-Bismut F, Ratziu V, Pieroni L, Charlotte F, Benhamou Y, Poynard $T$, for the MULTIVIRC group. Biochemical markers of liver fibrosis in patients wich hepatitis $C$ virus infection: a prospective study. Lancet 2001; 357:1069-1075. 
32. Merkel C, Bolognesi M, Finucci GF, Angeli P, Caregaro L, Rondana M, Gatta A. Indocyanine green intrinsic hepatic clearance as a prognostic index of survival in patients with cirrhosis. J Hepatol 1989;9:16-22.

33. Herold C, Heinz R, Niedobitek G, Schneidet T, Hahn EG, Schuppan D. Quantitative testing of liver function in relation to fibrosis in patients with chronic hepatitis B and C. Liver 2001;21:260-526.

34. Aube C, Oberti F, Korali N, Namour MA, Loisel D, Tanguy JY, Valsesia $\mathrm{E}$, et al. Ultrasonographic diagnosis of hepatic fibrosis or cirrhosis. J Hepatol 1999;30:472-478
35. Albrecht T, Blomley MJ, Cosgrove DO, Taylor-Robinson SD, Jayaram V, Eckersley R., Urbank A, et al. Non-invasive diagnosis of hepatic cirrhosis by transit-time analysis of an ultrasound contrast agent. Lancet 1999;353: $1579-1583$.

36. Hoefs JC, Wang F, Kanel G. Functional measurement of nonfibrotic hepatic mass in cirrhotic patients. Am J Gastroenterol 1997;92:20542058.

37. Grace N. Diagnosis and treatment of gastrointestinal bleeding secondary to portal hypertension. Am J Gastroenterol 1997:92:1081-1091. 